\title{
Aspectos clave en el mantenimiento de la diuresis residual en pauta de hemodiálisis incremental. Experiencia de diez años
}

\author{
Esther García, José Luis Merino, Sonia Mendoza, Vicente Paraíso, Verónica Sánchez, Raquel Moya \\ Sección Nefrología. Hospital Universitario del Henares. Madrid. España
}

Como citar este artículo:

García E, Merino JL, Mendoza S, Paraíso V, Sánchez V, Moya R.

Aspectos clave en el mantenimiento de la diuresis residual en pauta de hemodiálisis incremental.

Experiencia de diez años

Enferm Nefrol. 2020 Abr-Jun;23(2):199-204

\section{Resumen}

Introducción: La hemodiálisis incremental o progresiva es una modalidad de inicio de hemodiálisis, basada en la diuresis residual y adaptada a las necesidades del paciente, poco extendida pese a sus potenciales beneficios. Para su correcto seguimiento es necesario establecer unas pautas específicas en cada sesión de hemodiálisis, que deben ser conocidas por el personal que atiende a estos pacientes de forma regular.

Objetivo: analizar la evolución de los pacientes que han iniciado tratamiento renal sustitutivo con hemodiálisis incremental.

Material y Método: Estudio observacional retrospectivo de pacientes incidentes en tratamiento renal sustitutivo mediante hemodiálisis incremental en nuestro centro en los últimos 10 años. Comparación de resultados basales y a los 12 meses de seguimiento.

Resultados: En este periodo de tiempo se han incluido 49 pacientes en técnica de hemodiálisis incremental. Aunque la diuresis residual desciende en el primer año de $2030 \pm 600 \mathrm{ml} / \mathrm{día}$ a $1300 \pm 500(p<0,05)$, ésta

\section{Correspondencia:}

Esther García-García

Email: esther.garcia@salud.madrid.org se mantiene por encima de un litro en la mayoría de los casos. El aclaramiento de urea también desciende de $5,7 \pm 1,6 \mathrm{ml} / \mathrm{min}$ a $3,4 \pm 1,6 \mathrm{ml} / \mathrm{min}$ al año $(p<0,05)$.

Conclusiones: Iniciar tratamiento renal sustitutivo con hemodiálisis incremental puede mantener más tiempo la diuresis residual, para eso es clave el conocimiento de la técnica y su correcto manejo durante las sesiones de diálisis.

PALABRAS CLAVE: diálisis incremental; diuresis residual; hemodiálisis; cuidados de enfermería.

\section{Key aspects in maintaining residual diuresis in an incremental haemodialysis schedule. Experience of ten years}

\section{Abstract}

Introduction: Incremental or progressive haemodialysis is a modality for starting haemodialysis, based on residual diuresis and adapted to the needs of the patient, and not very widespread despite the potential benefits. For correct follow-up, it is necessary to establish specific guidelines in each haemodialysis session, which must be known by the staff who treat these patients regularly. 
Aim: To analyse the evolution of patients who start renal replacement therapy with incremental haemodialysis.

Material and Method: Retrospective observational study of incident patients on renal replacement therapy using incremental haemodialysis in our centre in the last 10 years. Comparison of baseline and 12-month follow-up results was carried out.

Results: In the study period, 49 patients with incremental haemodialysis were included. Although the residual diuresis falls in the first year from $2030 \pm 600$ $\mathrm{ml} /$ day to $1300 \pm 500(p<0.05)$, in most cases, it remains above one litre. Urea clearance also decreases from $5.7 \pm 1.6 \mathrm{ml} / \mathrm{min}$ to $3.4 \pm 1.6 \mathrm{ml} / \mathrm{min}$ per year $(p<0.05)$.

Conclusions: Starting renal replacement therapy with incremental haemodialysis can keep residual diuresis longer. Knowledge of the technique and correct handling during dialysis sessions are key.

KEYWORDS: incremental dialysis; residual urine; haemodialysis; nursing care.

\section{Introducción}

La hemodiálisis incremental (HDI), con 2 sesiones a la semana, como pauta de inicio de tratamiento renal sustitutivo (TRS) se basa en el mantenimiento de la diuresis residual $(D R)$ y en su ajuste progresivo según desciende la función renal residual (FRR) ${ }^{1}$. Es bien conocido que la DR en la hemodiálisis (HD) convencional se pierde más rápidamente que en otras técnicas como la diálisis peritoneal (DP), donde preservar la FRR es un aspecto prioritario ${ }^{2}$. Es por ese motivo que, en general, la DR se valora en menor medida que en HD convencional. No obstante, una adecuada DR en pacientes con enfermedad renal crónica en TRS, potencialmente, aporta beneficios sobre aquellos pacientes que están en situación de anuria ${ }^{3}$. Por eso, independientemente de la modalidad que se opte por TRS, la DR debería ser un objetivo para preservar ${ }^{4}$. Para conservar la DR, entre otros aspectos, es crucial un adecuado control en la sesión de HD, con el objetivo de evitar situaciones de inestabilidad hemodinámica. Evitar episodios de hipotensión arterial y realizar un seguimiento estrecho del teórico peso seco, evitando tasas de ultrafiltración elevadas, son aspectos clave bajo el control de enfermería ${ }^{5,6}$. Actualmente la pauta de hemodiálisis tiende a optimizarse y a individualizarse para cada paciente, de acuerdo con diferentes características y expectativas vitales. La presencia de una adecuada DR puede aportar una serie de beneficios, como un mejor control de volumen, una mayor eliminación de medianas moléculas, una mejor calidad de vida e incluso una mayor supervivencia en algunas series publicadas ${ }^{7,8}$. Esta DR es clave en la modalidad de TRS de DP, en este tipo de pacientes está claramente demostrada su utilidad y es prioritario su cuidado 9 . En HD, con pautas adaptadas, como la HDI, de inicio dos sesiones de HD a la semana también se pude preservar la DR y ofrecer, al igual que en $D P$, esos beneficios a nuestros pacientes ${ }^{10}$.

Desde la apertura de nuestra unidad de HD la pauta de HDI es una alternativa para nuestros pacientes. EI equipo de enfermería es clave para alcanzar los beneficios buscados con esta pauta, manteniendo una especial vigilancia en las sesiones de HD y asegurando una educación adecuada de los pacientes en HDI ${ }^{11,12}$. Desde marzo 2008 hasta marzo 2017, mostramos los resultados de su aplicación en nuestro hospital, con el objetivo de evaluar la efectividad de la HDI como modalidad de inicio del TRS.

\section{Material y Método}

Estudio observacional retrospectivo de los pacientes incidentes que comenzaron TRS en pauta de HD incremental en el Hospital Universitario del Henares, Madrid, desde marzo de 2008 hasta marzo de 2017. Se incluyeron en el estudio todos los pacientes que iniciaron HDI en nuestro centro. Se consideraron criterios para indicación de HDI los siguientes: presentar una diuresis residual de al menos $1000 \mathrm{ml} / 24 \mathrm{~h}$, encontrase en situación de estabilidad clínica, ausencia de edemas, no evidencia de hiperpotasemia $>6,5 \mathrm{mEq} / \mathrm{L}$, no fosforemia $>6 \mathrm{mg} / \mathrm{dl}$, y que se evidenciase una aceptable comprensión de los cuidados dietéticos. Se consideraron como criterios de exclusión un mal cumplimiento dietético o farmacológico. Los pacientes con menos de seis meses de permanencia en HDI, tanto por recuperación de función renal residual, como por necesidad de incrementar a tres sesiones de HD fueron excluidos del análisis estadístico.

Los criterios para pasar de HDI a pauta de HD convencional ( 3 sesiones de HD/semana) fueron: presentar una $\mathrm{DR}<1000 \mathrm{ml} / 24 \mathrm{~h}$ en al menos dos controles, un aclaramiento de urea $<2,2 \mathrm{ml} / \mathrm{min}$, un mal control de potasio, fósforo o acidemia, un no óptimo control de TA prediálisis (TA $>160 / 95 \mathrm{~mm} \mathrm{Hg}$ de forma persistente) 0 episodios de insuficiencia cardiaca o sobrecarga hidrosalina. 
Como variables del estudio se recogieron la edad media de los pacientes al inicio de la HDI, tiempo de seguimiento en dicha modalidad, diuresis residual, ganancia de peso interdiálisis, tasa de UF horaria durante la sesión de HD, así como los niveles séricos de potasio, fósforo y el estado de acidosis, evaluado este último mediante los niveles de bicarbonato en sangre. En aquellos pacientes que hubo que trasferir de HDI a HD convencional también se recogieron los motivos de este cambio.

La diuresis residual se estudió bimensualmente, con la recogida de orina de 24 horas el día previo a la sesión de HD. El resto de parámetros analíticos se evaluaban mensualmente. Por otra parte, cuatrimestralmente, se realizaba un estudio mediante bioimpedancia para analizar el componente de agua libre, lo que ayudaba a estimar el peso seco, asegurando preservar la $D R$, pero no a costa de una sobrehidratación contraproducente. En ese sentido el seguimiento de la TA debía ser exhaustivo, vigilando aquellos pacientes con mayor dificultad en su control prediálisis y durante la sesión de HD.

Tabla 1. Principales aspectos de control por parte de enfermería en el manejo de pacientes con pauta de hemodiálisis incremental (HDI).

Aspectos claves de Control por Enfermería en HDI

1. Considerar el peso seco como relativo.

2. Evitar tasa de ultrafiltración $>500 \mathrm{ml} / \mathrm{h}$.

3. Minimizar los episodios de hipotensión arterial intradiálisis.

4. Vigilar la TA prediálisis.

5. Recomendar hábitos dietéticos.

6. Controlar la evolución de la diuresis residual.

7. Explorar la presencia de edemas y sobrecarga hídrica.

8. Realizar bioimpedancia periódica.

En la Tabla 1 se muestran los aspectos claves que el equipo de enfermería debería controlar en este tipo de pacientes. El peso seco era considerado como orientativo, en ese sentido, el personal de enfermería programaba tasas de $U F<500 \mathrm{ml} / \mathrm{h}$ y reevaluaba la necesidad de plantear un ajuste en base al resto de parámetros. Se analizaba en cada sesión la presencia de edemas, los datos de sobrecarga hidrosalina, la TA prediálisis y si se preguntaba por la diuresis, así como se avisaba si el incremento del teórico peso era mayor de los $2 \mathrm{Kg}$ de ganancia interdiálisis. También se trasmitía la importancia de la DR, con un control de esta, con una correc- ta recogida periódicamente, para su evaluación y así adecuar la pauta de HDI continuamente. No obstante, pese a la $D R$, estos pacientes debían seguir recomendaciones dietéticas adaptadas a su situación. Si bien dicha DR permitía una ingesta hídrica más laxa, esta no debe ser infravalorada, así como tampoco la vigilancia de los niveles de potasio y del metabolismo calcio-fósforo que, aun siendo más flexible en esta pauta, tampoco debe despreciarse en su control.

La información recogida ha sido tratada con arreglo a la actual Ley Orgánica 3/2018, de 5 de diciembre de protección de datos personales y garantía de los derechos digitales y con el único fin del desarrollo de esta investigación.

Respecto al análisis estadístico, se realizó un análisis descriptivo, donde se presentaron las variables cuantitativas mediante medias y desviación estándar en caso de normalidad y mediana y rango en caso de no normalidad. Los datos analizados tienen una distribución normal, por lo que se utilizaron test paramétricos. Se ha utilizado la prueba $T$ de Student para datos pareados para la comparación de medias. Un valor de $p<0,05$ fue considerado estadísticamente significativo.

\section{Resultados}

Durante el periodo de estudio, un total de 49 pacientes fueron incluidos en el programa de HDI, de los que sólo 28 conformaron la muestra final estudiada (ver Figura 1). La edad media al inicio de TRS era de $63 \pm 12$ años. La distribución por sexos fue de 8 mujeres y 20 varones. La enfermedad de base para iniciar diálisis fue: diabetes mellitus 7 pacientes, glomerulonefritis 6 pacientes, nefroangiosclerosis 2 pacientes, nefropatía intersticial 2 pacientes, poliquistosis renal 2 pacientes, otras $5 \mathrm{pa}$ cientes y no filiadas en 4 pacientes. La permanencia media en TRS fue de $24 \pm 21$ meses (rango: 6-74 meses), con un tiempo medio en técnica incremental de 16 \pm 18 meses (rango: 6-74 meses).

La diuresis residual descendió en el primer año de 2030 $\pm 600 \mathrm{ml} / \mathrm{día}$ a $1300 \pm 500(p<0,05)$. El aclaramiento de urea también descendió de $5,7 \pm 1,6 \mathrm{ml} / \mathrm{min}$ a $3,4 \pm 1,6$ $\mathrm{ml} / \mathrm{min}$ al año $(p<0,05)$. El peso medio seco fue ajustándose a lo largo de los meses, el peso basal fue de $72 \pm 14 \mathrm{Kg}$ y de $70,6 \pm 14$ y $64 \pm 14$, a los seis y doce meses, respectivamente. El descenso porcentual a los 6 meses fue de un $2 \%$ y de un $22 \%$ a los doce meses, respecto al control basal. 


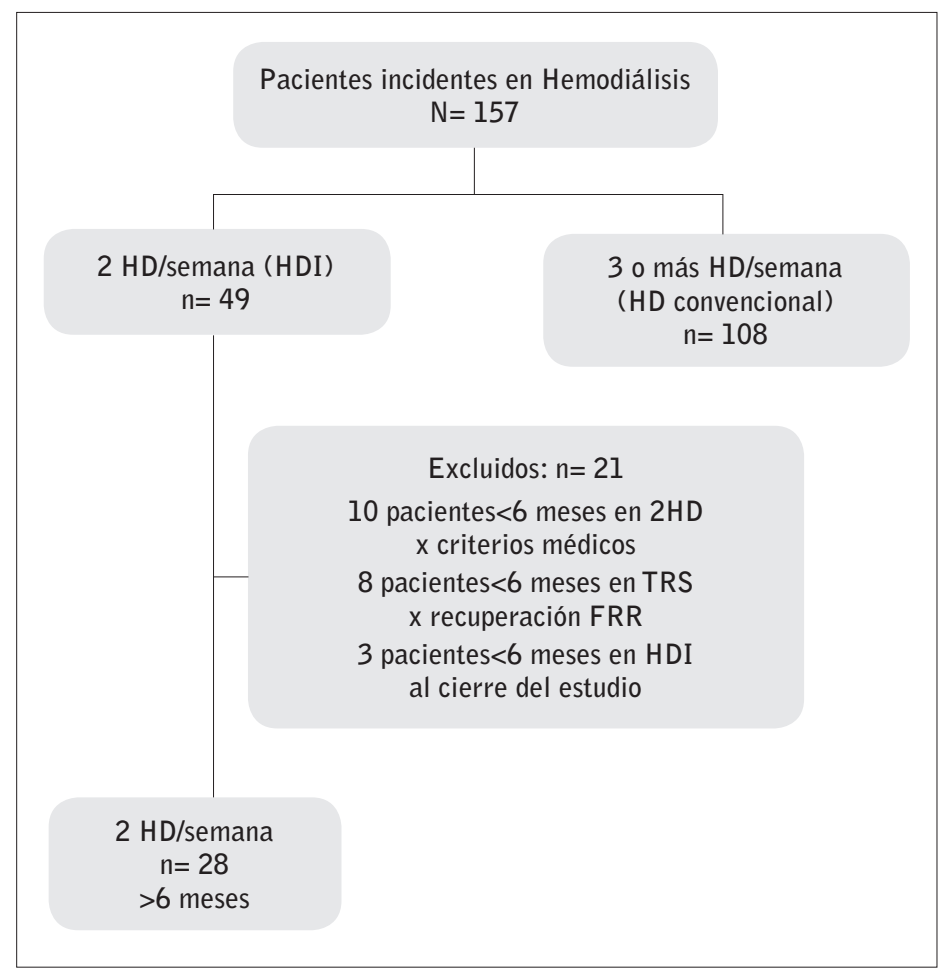

Figura 1. Pacientes incidentes en hemodiálisis desde marzo 2008 a marzo 2017. HD: hemodiálisis. TRS: tratamiento renal sustitutivo, FRR: función renal residual, HDI, hemodiálisis incremental.

Las principales causas de pasar de 2 a 3 sesiones de HD por semana fueron la presencia de alteraciones en los parámetros analíticos (hiperpotasemia, acidosis, descenso del aclaramiento de urea), los episodios de sobrecarga de volumen, con necesidad de reducir el peso seco, sesiones de HD repetidas con mala tolerancia, evidencia de mala adherencia terapéutica, así como el recibir un trasplante renal. Solo un paciente falleció bajo esta pauta de HDI.

\section{Discusión}

La pauta de HDI, 2 HD semana, ajustada de forma individual puede mantener más tiempo la diuresis residual. En nuestra serie se mantiene por encima de un litro al menos durante el primer año. En el estudio de Fernández-Lucas et al, con un seguimiento a 5 años, observaron resultados similares, con una mayor supervivencia de aquellos pacientes que mantuvieron más tiempo la diuresis residual ${ }^{13}$. Para poder conseguir una pauta con estas características es clave que los profesionales encargados de las sesiones de HD sean conscientes de la misma y conozcan los aspectos críticos para su control.

Lin et al en su trabajo de 2009, donde comparaban 23 pacientes en HDI vs 53 en HD convencional observaron resultados semejantes a los 18 meses de seguimiento, con una preservación estadísticamente significativa de la DR en pauta de HDI en comparación con $\mathrm{HD}$ convencional de $3 \mathrm{HD} /$ semana $^{14}$. Estos autores describían que el menor número de hipotensiones justificaría en parte sus resultados. En nuestra serie se ajustaba el peso seco progresivamente y se descendía paulatinamente a lo largo del seguimiento, dado el ajuste que requieren nuestros pacientes una vez en TRS. Es bien conocido que los episodios de hipotensión arterial son uno de los elementos críticos en la pérdida de FRR y por lo tanto evitarlos es crucial ${ }^{15}$. EI equipo de enfermería que controla las sesiones de HD debe tener en cuenta este aspecto clave, asegurando, en lo posible, que acontezcan en la menor medida. Por eso, el peso seco o su ganancia interdiálisis debe ser considerado diferente al paciente en HD 3 veces por semana en situación de anuria.

En el control diario se establecieron diversas indicaciones para este tipo de pacientes, tanto en las sesiones de diálisis habituales como en el seguimiento periódico mensual. Así, el peso seco en este tipo de pacientes es relativo, evitando tasas excesivas de ultrafiltración con el objetivo de evitar hipotensiones, como factor crítico para deteriorar la $\mathrm{FRR}^{16}$. Este peso seco teórico debe ser un objetivo relativo y siempre bajo las premisas de evitar alta tasas de ultrafiltración, que es el principal factor de riesgo para provocar hipotensión arterial como ya se ha demostrado en diversos estudios ${ }^{17}$. Esta tasa máxima no debía exceder los $500 \mathrm{ml} / \mathrm{h}$, reevaluando en cada sesión la presencia de edemas y la tensión arterial (TA). Inicialmente el peso seco se ajustaba según tolerancia, TA y parámetros analíticos, introduciendo, a lo largo de los años, el control de este mediante bioimpedancia. Paralelamente cada dos meses era reevaluada la DR y la FRR en control analítico, mediante la recogida de orina de 24 horas. También todos los pacientes recibían indicaciones dietéticas, consejos sobre ingesta hídrica, así como sobre el control de la TA.

Desafortunadamente, existen escasos estudios centrados en HDI con suficiente poder estadístico. Así, Liu y colaboradores, en su metaanálisis de 2019 donde incluyeron 16 estudios, quince de ellos observacionales, evidenciaron que la pauta de HDI ofrece una mayor supervivencia y además mantiene más tiempo la diuresis residual, al menos durante el primer año, tal y como refleja también nuestra serie de 10 años de seguimiento ${ }^{11}$. 
No obstante, esta pauta debe ser reconsiderada y evaluada periódicamente con el objetivo de evitar complicaciones, especialmente la situación de sobrehidratación, el mal control de TA o las complicaciones iónicas propias del paciente renal. En ese sentido, enfermería debería tener conocimiento de la pauta de HDI, en cuanto a sus beneficios y a su manejo en la práctica clínica diaria. Una pauta optimizada y ajustada de forma segura, bajo personal especializado, puede aportar beneficios, más allá de unos conocidos a corto plazo como otros más trascendentales, como una mayor supervivencia y una mejor calidad de vida, todavía por demostrar. Hasta que se consigan unos estudios clínicos aleatorizados con suficiente potencia estadística y se puedan obtener conclusiones firmes, el desarrollo de estos estudios observacionales pueden aportar información útil en la práctica clínica habitual, asumiendo los posibles sesgos de selección y las limitaciones propias de este tipo de estudios.

El control del peso seco, la tasa de ultrafiltración, la tensión arterial y su vigilancia durante la sesión de HD son aspectos clave bajo la supervisión del equipo de enfermería. Así, a partir de nuestros resultados, los pacientes en HDI preservan la función renal residual, especialmente durante el primer año. Esta modalidad de 2 sesiones de HD por semana puede ser una alternativa en un grupo seleccionado de pacientes.

Recibido: 01-02-20

Revisado: 15-02-20

Modificado: 23-02-20

Aceptado: 30-02-20

\section{Bibliografía}

1. Wong J, Vilar E, Davenport A, Farrington K. Incremental haemodialysis. Nephrol Dial Transplant. 2015;30(10):1639-48.

2. Selby NM, Kazmi I. Peritoneal dialysis has optimal intradialytic hemodynamics and preserves residual renal function: Why isn't it better than hemodialysis? Semin Dial. 2019;32(1):3-8.
3. Vilar E, Wellsted D, Chandna SM, Greenwood RN, Farrington K. Residual renal function improves outcome in incremental haemodialysis despite reduced dialysis dose. Nephrol Dial Transplant. 2009;24(8):2502-10.

4. Rodríguez-Benítez P, Gómez-Campderá FJ. Importance of the residual renal function in hemodialysis patients. Nefrologia. 2002;22(2):98-103.

5. National Kidney Foundation: K/DOQI Clinical Practice Recommendations for Hemodialysis Adequacy. Guideline 6: Preservation of residual kidney function. Am J Kidney Dis 2006;48(Suppl 1):S68-70.

6. Kalantar-Zadeh K, Unruh M, Zager PG, Kovesdy CP, Bargman JM, Chen J, et al. Twice-weekly and incremental hemodialysis treatment for initiation of kidney replacement therapy. Am J Kidney Dis. 2014;64(2):181-6.

7. Vanholder R, Van Biesen W, Lameire N. Is starting hemodialysis on a twice-weekly regimen a valid option? Am J Kidney Dis. 2014;64(2):165-7.

8. Chin AI, Appasamy S, Carey RJ, Madan N. Feasibility of Incremental 2-Times Weekly Hemodialysis in Incident Patients With Residual Kidney Function. Kidney Int Rep. 2017;2(5):933-42.

9. Nongnuch A, Assanatham M, Panorchan K, Davenport $A$. Strategies for preserving residual renal function in peritoneal dialysis patients. Clin Kidney J. 2015;8(2):202-11.

10. Liu Y, Zou W, Wu J, Liu L, He Q. Comparison between incremental and thrice-weekly haemodialysis: Systematic review and meta-analysis. Nephrology (Carlton). 2019;24(4):438-44.

11. Kuipers J, Oosterhuis JK, Krijnen WP, Dasselaar JJ, Gaillard CA, et al. Prevalence of intradialytic hypotension, clinical symptoms and nursing interventions--a three-months, prospective study of 3818 haemodialysis sessions. BMC Nephrol. 2016;17:21.

12. Zhang M, Wang M, Li H, Yu P, Yuan L, Hao C, et al. Association of Initial Twice-Weekly Hemodialysis Treatment with Preservation of Residual Kidney Function in ESRD Patients. Am J Nephrol. 2014; 40(2):140-50. 
13. Fernández-Lucas M, Teruel-Briones JL, Gomis-Couto A, Villacorta-Pérez J, Quereda-Rodríguez-Navarro $\mathrm{C}$. Maintaining residual renal function in patients on haemodialysis: 5-year experience using a progressively increasing dialysis regimen. Nefrologia. 2012;32(6):767-76.

14. Lin YF, Huang JW, Wu MS, Chu TS, Lin SL, Chen $Y M$, et al. Comparison of residual renal function in patients undergoing twice-weekly versus three-times-weekly haemodialysis. Nephrology (Carlton). 2009;14(1):59-64.

Este artículo se distribuye bajo una Licencia Creative Commons Atribución-NoComercial 4.0 Internacional. https://creativecommons.org/licenses/by-nc/4.0/

\section{Open Access (c) () (8)}

